Cahiers $d u$ MONDE RUSSE

\section{Cahiers du monde russe}

Russie - Empire russe - Union soviétique et États indépendants

$46 / 4 \mid 2005$

L'invention d'une politique humanitaire

\title{
Action publique, action humanitaire pendant le premier conflit mondial
}

Les zemstvos et les municipalités

\section{OLGA PICHON-BOBRINSKOY}

\section{(2) OpenEdition}

Journals

Édition électronique

URL : https://journals.openedition.org/monderusse/9423

DOI : $10.4000 /$ monderusse. 9423

ISSN : $1777-5388$

Éditeur

Éditions de l'EHESS

Édition imprimée

Date de publication : 1 décembre 2005

Pagination : 673-698

ISBN : 2-7132-2057-2

ISSN : $1252-6576$

Référence électronique

OLGA PICHON-BOBRINSKOY, «Action publique, action humanitaire pendant le premier conflit mondial », Cahiers du monde russe [En ligne], 46/4 | 2005, mis en ligne le 01 janvier 2005, consulté le 04 septembre 2022. URL : http://journals.openedition.org/monderusse/9423 ; DOI : https://doi.org/ 10.4000 /monderusse. 9423 


\section{ACTION PUBLIQUE, ACTION HUMANITAIRE PENDANT LE PREMIER CONFLIT MONDIAL}

\section{Les zemstvos et les municipalités}

Acteurs à part entière de la politique de modernisation lancée par les Grandes Réformes, les self-gouvernements locaux furent l'un des grands vecteurs de la promotion et de la mise en œuvre de l'action publique, action qui se situait en dehors du champ gouvernemental, mais cependant au service de l'intérêt général ${ }^{1}$. Le caractère électif, mais aussi la fonction de gestion locale des services publics assignée par l'État central conférèrent aux zemstvos et aux municipalités un caractère privilégié, propice à l'émergence de cette notion. La nouvelle société civile trouvait son identification dans le service aux populations et l'engagement dans les affaires publiques et y voyait l'une des expressions de son aspiration réformatrice ${ }^{2}$.

Sous la pression des grandes crises nationales que furent la guerre russo-japonaise et les famines de 1905, 1906 et 1907, les organes d'auto-administration investirent un champ amené à se développer, le champ humanitaire. Jusqu'alors circonscrite à la sphère locale par les autorités tsaristes, cette nouvelle orientation signifia un changement d'échelle pour l'action publique des zemstvos et des municipalités, posant ainsi les jalons de leur expansion au cours de la Grande Guerre. En effet, avec le déclenchement de celle-ci, le champ d'intervention des self-gouvernements regroupés en Unions des zemstvos et des villes s'étendit alors de l'action humani-

1. Thomas Porter, William Gleason, «The Zemstvo and Public Initiative in Late Imperial Russia », Russian History, 21 (4), 1994, p. 419-437 ; Terence Emmons et Wayne S. Vucinich, eds., The Zemstvo in Russia : An Experiment in Local Self-Governement, Cambridge, NewYork : Cambrigde University Press, 1982 ; P. Gronsky, N. Astrov, The War and the Russian Government, New Haven, 1929 ; Joseph Bradley, Muzhik and Muscovite : Urbanization in Late Imperial Russia, Berkeley : University of California Press, 1985.

2. E. I. Kimerling Wirtshafter, Social Identity in Imperial Russia, DeKalb : Northern Illinois University Press, 1997. En particulier, le chapitre III, « Middle Groups », p. 63-99. 
taire à une politique plus vaste de partenariat avec les autorités civiles et militaires pour la gestion des conséquences du conflit.

Le présent article a une double finalité : établir la place de l'action humanitaire dans les mutations d'une action publique placée sous le signe de l'extension de son champ de compétences et donc établir son rôle comme mode de légitimation de la montée en puissance des Unions au cours de la guerre. Mais, en retour, il s'intéresse aussi à l'influence de cette montée en puissance sur les pratiques humanitaires elles-mêmes, l'accroissement et la diversification des actions des Unions dans ce domaine les amenant, en effet, à concevoir de nouvelles méthodes de gestion et de planification.

L'étude de l'action des Unions en faveur des réfugiés devrait permettre de suivre le processus de transformation d'une action d'assistance en un problème politique et gestionnaire. La politisation de l'action humanitaire constitue ainsi l'un des aspects du glissement qui s'opère, au cours de la guerre, d'une action publique conçue non plus en partenariat mais en substitution de l'État.

\section{À l'origine de l'action humanitaire en Russie : les catastrophes nationales (1904-1914)}

La première décennie du $\mathrm{Xx}^{\mathrm{e}}$ siècle constitua pour les gouvernements locaux d'auto-administration, et les zemstvos en particulier, une période d'expérimentation d'un genre nouveau : l'action d'assistance à l'échelle locale, mais aussi nationale, aux populations victimes de guerres et de calamités. En mars 1904, dès le début du conflit russo-japonais, 13 zemstvos de provinces se regroupèrent et prirent le nom d'Organisation générale des zemstvos (Obščaja zemskaja organizacija $^{3}$. Le but de cette union était de financer et d'organiser l'envoi d'équipes médicales en Mandchourie, pour venir en aide aux soldats blessés. C'est sur fond de mobilisation politique des zemstvos entraînés dans le Mouvement de libération (Osvoboditel'noe dviženie) que, pour la première fois, le gouvernement impérial autorisa les zemstvos à agir de façon concertée et, qui plus est, sur des territoires sortant de leur juridiction. Cette initiative, dont la finalité était exclusivement humanitaire, constitua la première étape d'un mouvement de rassemblement des zemstvos au niveau national, mouvement qui trouva son apogée avec la tenue de grands congrès des représentants des zemstvos, fin 1904 et tout au long de l'année $1905^{4}$.

Fondée par des organismes publics, mais placée officiellement sous l'égide de la Croix-Rouge russe, l'Organisation générale des zemstvos trouvait ainsi, dans le cadre associatif privé, le moyen de mener une action qu'elle concevait comme

3. T. I. Polner, Obščezemskaja organizacija na dal'nem vostoke, Moscou, 1908-1910 ; Porter, Gleason, « The Zemstvo and Public Initiative... » p. 421-424.

4. Liberal'noe dviženie v Rossii : 1902-1905, M. : ROSSPEN, 2001. 
publique, dans la mesure où il s'agissait de combler les carences de l'État dans le domaine de la gestion sanitaire de la guerre 5 .

Les zemstvos participant à l'action d'assistance aux soldats russes blessés en Extrême-Orient s'étaient engouffrés au sein de l'armée dans une brèche provoquée par la profonde désorganisation des opérations de secours. Cette situation fut à l'origine de l'appel, fin février 1904, du président du Comité exécutif de la CroixRouge russe, le comte Voroncov-Daškov aux zemstvos et aux doumas municipales pour qu'ils lui viennent en aide ${ }^{6}$. En mars 1904, les représentants de 19 zemstvos de province firent parvenir une proposition de participation à la mise en place d'équipes médico-sanitaires, sous réserve que la coordination de l'action des zemstvos serait assurée via une nouvelle organisation, l'Organisation générale des zemstvos. Dans un premier temps, le ministre de l'Intérieur Plehve multiplia les obstacles à la mobilisation concertée des zemstvos, y voyant une entrave à l'article $3 \mathrm{du}$ statut du zemstvo qui interdisait toute action de cette institution en dehors de son territoire ${ }^{7}$. En février 1904, pour contourner l'opposition de la bureaucratie, l'Organisation générale des zemstvos représentée par le prince Georgij L'vov, président du directoire du zemstvo de Tula, s'adressa directement à Nicolas II, lui demandant de lever les entraves faites aux zemstvos qui s'étaient ralliés à l'Organisation. La réponse favorable du tsar, sans mettre complètement fin à toutes ces difficultés, permit de relancer la mobilisation des zemstvos au sein de l'Organisation ${ }^{8}$. La loi sur l'interdiction aux zemstvos d'entreprendre des actions en commun resta néanmoins en vigueur, et ce n'est que le 6 octobre 1905 que le ministère de l'Intérieur donna une sanction officielle à l'existence de l'Organisation. Entre temps, celle-ci avait déjà collecté auprès des zemstvos plus de 2 millions de roubles, équipé 21 unités médicales, envoyé 360 personnes (dont 43 médecins et 100 infirmières), mis en place 2 hôpitaux à Harbin, fourni un traitement médical à 50835 soldats et distribué des repas chauds à 389579 soldats ${ }^{9}$.

5. Sur la collaboration entre la Croix-Rouge et l'Organisation générale des zemstvos sur le front d'Extrême-Orient, voir T. I. Polner, Žiznennyj put' knjazja G. E. L'vova, P., 1932, p. 71-73.

6. Polner, Žiznennyj put' ..., p. 66. Polner, qui fut l'un des organisateurs de l'expédition de l'Organisation générale des zemstvos en Extrême-Orient, explique l'appel de la Croix-Rouge russe à la collaboration des zemstvos par la forte baisse de sa popularité et donc de ses sources de financement, ce qui l'empêchait, selon lui, de mener une action d'assistance de grande envergure. Ibid., p. 68.

7. Ibid. Les gouverneurs reçurent l'ordre d'empêcher toute future tentative de ralliement des zemstvos à l'Organisation. L'annulation des assignations votées par les assemblées constitue un exemple de ces obstacles.

8. À ses débuts, l'Organisation générale des zemstvos n'avait pas de véritable organe directeur. Le siège, situé à Moscou, était dirigé par un délégué général, Dmitrij Šipov, entouré des membres de l'uprava du zemstvo de la province de Moscou. Quant au travail opérationnel en Mandchourie, il était coordonné par un autre délégué général, le prince G. E. L'vov. À l'occasion du congrès national des zemstvos du 2 mars 1905, les zemstvos membres de l'Organisation élirent un comité exécutif permanent composé de D. Šipov, G. E. L'vov, M. V. Čelnokov, F. A. Golovin et N. N. Hmelev. Polner, Žiznennyj put' ..., p. 130-131.

9. Cité par Porter, Gleason, « The Zemstvo and Public Initiative... », p. 423. 
Le spectre d'une nouvelle famine qui menaçait 23 provinces des régions du Centre et de la Volga conduisit l'Organisation générale des zemstvos à prolonger son existence au-delà du conflit russo-japonais. Cette fois-ci, la volonté de mobilisation de l'Organisation pour cette nouvelle crise rencontra immédiatement les encouragements des autorités gouvernementales particulièrement démunies devant l'ampleur de l'imminente catastrophe. Le gouvernement manifestait ici un véritable retour en arrière par rapport à l'orientation qu'il avait prise depuis 1900 en empêchant les zemstvos de jouer un rôle actif dans la prévention des famines. Ainsi, le décret de juin 1900, qui retirait aux zemstvos la gestion des subsistances, fut suspendu et, le 6 décembre 1905, une circulaire du ministère de l'Intérieur ordonna aux gouverneurs de faciliter le travail entrepris par l'Organisation dans leurs provinces. Contrairement à l'action en faveur des soldats, dont le poids financier avait reposé essentiellement sur les zemstvos, le financement de la lutte contre la famine fut assuré en majeure partie par l'État, soit par le biais de subventions directes à l'Organisation ou par celui de subventions aux zemstvos, reversées ensuite à l'Organisation par les zemstvos membres ${ }^{10}$. L'année 1906-1907 qui vit arriver une nouvelle famine marqua une étape supplémentaire dans la volonté gouvernementale de collaborer avec l'Organisation générale des zemstvos. Celle-ci fut invitée, aux côtés de la Croix-Rouge russe et de l'organisation caritative Trudovaja pomošč (Aide par le travail), à envoyer un délégué au Comité central d'aide médicale et alimentaire (Central'nyj komitet po okazaniju vračebno-pitatel'noj pomošči), organe intergouvernemental chargé de la répartition des subsides gouvernementaux dans la lutte contre la famine ${ }^{11}$.

Devenue un organisme permanent dont la vocation était de répondre à des situations d'urgence, l'Organisation générale des zemstvos dut trouver sa place et sa légitimité dans un champ humanitaire qui mobilisait à la fois les acteurs du public et du privé. L'engagement de la société civile étant resté limité lors du conflit russojaponais, l'action de l'Organisation générale des zemstvos en faveur des soldats russes mobilisés, bien que modeste en moyens et en hommes, avait été particulièrement visible et l'avait immédiatement portée sur le devant de la scène publique. La famine qui, cette fois-ci, touchait de nombreuses provinces de l'empire et survenait dans un contexte social et politique explosif, avait une résonance autrement importante dans la société. Ceci justifiait l'ambition exprimée au cours du congrès des zemstvos de juin 1905 de jouer un rôle fédérateur de la mobilisation humanitaire de l'obščestvennost':

C'est à cette même organisation publique qui a déjà coordonné le travail des équipes médicales des zemstvos en Extrême-Orient que doit revenir la responsa-

10. Les assemblées des zemstvos avaient participé à raison de 1036000 roubles pour l'envoi d'équipes médicales. T. I. Polner, Ob̌̌čezemskaja Organizacija..., t. 1, p. 51. En 1905-1906, l'État versa 208 millions de roubles aux zemstvos pour leur action contre la famine. Porter, Gleason, « The Zemstvo and Public Initiative... », p. 426.

11. Polner, Žiznennyj put’ ..., p. 135. 
bilité de l'aide publique et caritative (obščestvenno-blagotvaritel'naja) aux populations touchées par les mauvaises récoltes ${ }^{12}$.

Cette aspiration à coordonner les initiatives émanant de la charité privée (blagotvoritel'nost') n'avait rien d'original pour un organisme issu des assemblées publiques locales. Les organes d'auto-administration et le monde associatif privé avaient une longue habitude de collaboration, notamment pour les questions relatives à la pauvreté et à l'assistance publique ${ }^{13}$. Les zemstvos et les doumas avaient ainsi largement recours aux acteurs privés, leur déléguant des tâches qui relevaient de leurs compétences. Toutefois l'Organisation ne parvint pas à jouer ce rôle organisationnel du champ humanitaire qu'elle ambitionnait, notamment en raison de la présence d'autres structures concurrentes. Ainsi, l'organisation caritative Trudovaja pomošč (Aide par le travail) qui, depuis 1899, avait lancé plusieurs campagnes successives de lutte contre la famine dans les régions de Russie touchées par les mauvaises récoltes, pouvait aussi se prévaloir de son expérience pour exercer un rôle moteur dans l'engagement du monde associatif contre la famine. Par ailleurs, depuis fin 1905, l'Organisation générale des zemstvos subissait les conséquences des violentes campagnes menées par les socialistes ainsi que par les libéraux les plus radicaux contre les zemstvos. Ces campagnes, provoquées notamment par le virage à droite de nombreux zemstvos suite aux émeutes paysannes de 1905 , avaient terni indirectement l'image de l'Organisation générale des zemstvos ${ }^{14}$. L'Union des unions (Sojuz sojuzov), en créant sa propre organisation de lutte contre la famine, «l'Organisation publique »(Obščestvennaja organizacija) n'avait pas l'intention de collaborer avec l'Organisation générale des zemstvos ${ }^{15}$.

Au cours des deux années de famine, l'Organisation générale des zemstvos s'orienta exclusivement vers des actions ponctuelles d'assistance aux populations victimes de la famine. Ainsi, lors de la campagne de 1906-1907, elle entretint 9711 cantines et boulangeries, distribua 120 millions de repas et 48,5 millions de rations alimentaires dont bénéficièrent 1676000 personnes $^{16}$. L'Organisation se distinguait ainsi de Trudovaja pomošč́ qui avait associé, quant à elle, opérations de secours et mise en œuvre d'importants projets dans le domaine du développement rural (travaux publics, aide au développement de l'artisanat rural, mise en place de systèmes de garde pour les enfants, etc. $)^{17}$.

12. Liberal'noe dviženie v Rossii...,p. 299.

13. G. N. Uljanova. Blagotvoritel'nost' v Rossijskoj imperii: XIX-načalo XX veka, M., 2005, p. 250-252. Adele Lindenmeyr, Poverty is not a Vice: Charity, Society and State in Imperial Russia, Princeton, 1996, p. 60-73.

14. Roberta T. Manning, « The Zemstvo and Politics, 1864-1914 », in Emmons et Vucinich, eds., The Zemstvo in Russia..., p. 134-147.

15. Polner, Žiznennyj put'..., p. 132.

16. Ibid., p. 136.

17. Lidenmeyr, Poverty is not a Vice..., p. 191-192. 
Ce choix de l'Organisation était en partie dicté par le fait qu'elle considérait son action comme complémentaire de la politique de développement rural menée par les zemstvos locaux. Ceci étant, comprendre l'environnement social et économique des personnes assistées figurait naturellement au nombre des préoccupations de l'Organisation générale des zemstvos, elle-même émanation des zemstovs. La campagne d'aide aux paysans migrants de Sibérie (pereselency), lancée par l'Organisation au début de 1908, donna l'occasion à ses participants de montrer leur attachement à l'idée d'une amélioration structurelle des conditions de vie des populations secourues. En mars et avril 1908, le prince L'vov, accompagné d'une équipe de 140 personnes, se rendit dans les provinces de Primorsk et de l'Amour et organisa une aide alimentaire et sanitaire sur les routes empruntées par les migrants ainsi que dans les villages d'accueil. Profitant d'une baisse momentanée de l'activité du personnel de l'expédition, l'un de ses responsables, Tihon Polner lança une campagne d'investigation sur la province de l'Amour, région placée sous régime militaire. Le but était d'établir un bilan, jusque-là inexistant pour cette région, sur le mouvement de colonisation (pereselenčeskoe delo) et donc de faciliter le transfert des paysans migrants ${ }^{18}$. À cet effet, des volontaires munis d'un questionnaire statistique partirent, dans des conditions souvent rocambolesques, enquêter dans différentes parties de la province. L'aventure prit fin au moment où le ministère de l'Intérieur donna l'ordre de confisquer les données recueillies par les enquêteurs ${ }^{19}$.

En jouant à la fois sur la coopération de l'État, sur la participation des zemstvos et de franges plus larges de la société civile, la direction de l'Organisation générale des zemstvos avait mis en place une construction fragile dont l'équilibre risquait d'être rompu en cas de désengagement de l'un des acteurs. Les premières difficultés apparurent en 1908, alors que l'Organisation cherchait à clarifier ses attributions et à se poser comme un organisme permanent ayant pour vocation de répondre aux situations de crise. Dans une nouvelle charte, elle déclarait vouloir venir en aide aux populations dans le besoin (incluant les victimes de la famine, les colons, etc.) et prendre le relais des zemstvos dans le cas où ceux-ci ne pourraient entreprendre séparément des actions qui exigeaient une coordination à l'échelle nationale ${ }^{20}$. Or, le décalage était croissant entre l'Organisation générale des zemstvos et les zemstvos, dominés par les forces politiques conservatrices depuis les élections de 1906-1907. À cette occasion, le prince L'vov, lui-même, avait perdu son mandat de président du directoire de la province de Tula. Le refus du nouveau président du directoire du zemstvo de Moscou, N. F. Rihter, de continuer à participer aux actions de l'Organisation, alors même que Moscou en avait été la fondatrice, constitue l'un des exemples les plus éloquents de cette prise de distance ${ }^{21}$. Du côté des autorités et, dans un contexte d'accalmie des catastrophes, l'Organisation ne pouvait plus compter sur le soutien quasi incondi-

18. Polner, Žiznennyj put'..., p. 141-146.

19. Ibid. Les auteurs de l'enquête réussirent toutefois à sauvegarder une copie de ces matériaux qui donna lieu à la publication, en 1909, d'un livre intitulé Priamur'e.

20. Porter, Gleason, « The Zemstvo and Public Initiative... », p. 427.

21. Polner, Žiznennyj put’..., p. 161. 
tionnel de Stolypin dont elle avait bénéficié jusque-là22 . L'épisode de l'enquête statistique en Extrême-Orient contribua certainement à l'instauration de ce nouveau climat de méfiance. Il faut aussi y voir un refus plus généralisé de laisser les agences non étatiques pénétrer de façon durable dans les campagnes. Ainsi, lors de la nouvelle famine de l'automne-hiver 1911-1912, l'Organisation générale des zemstvos, mais aussi Trudovaja pomošč ainsi que d'autres organisations furent quasiment écartées de l'action d'assistance aux affamés ${ }^{23}$. Comme le répondit le président du Conseil des ministres, V. N. Kokovcev, aux représentants d'une vingtaine d'organisations venus lui demander l'autorisation d'entreprendre des actions d'aide aux affamés, il ne pouvait être question que les organisations publiques (ob̌̌čestvennye) puissent agir indépendamment de l'État ${ }^{24}$.

Le raidissement manifesté ici par les autorités devant une société civile dont l'action humanitaire était devenue, à la veille de la guerre, l'un des modes d'expression favoris, constitua la toile de fond dans laquelle devait s'inscrire la mobilisation des élites sociales au cours de la Grande Guerre.

\section{La Grande Guerre et les mutations de l'action publique}

L'ampleur de la catastrophe qui s'annonçait avec l'entrée de la Russie en guerre entraîna une forte mobilisation de l'ensemble des acteurs étatiques, publics et privés dans la gestion des conséquences du conflit. Se sachant concernés au premier chef par les pertes humaines qui marqueraient les prochains combats, les zemstvos, puis les municipalités optèrent pour la mise en place de structures centralisatrices des actions menées par les self-gouvernements locaux pour venir en aide à l'armée dans une de ses tâches les plus urgentes : l'organisation de l'évacuation des soldats malades et blessés vers l'arrière. Devenue une coquille vide depuis 1912, l'Organisation générale des zemstvos ne pouvait prétendre exercer le rôle de fédérateur des zemstvos dans les actions d'assistance. C'est donc une nouvelle structure qui se mit en place le 30 juillet 1914, l'Union des zemstvos de Russie d'aide aux soldats malades et blessés (Vserossijskij zemskij sojuz pomošči bol'nym i ranenym voinam $)^{25}$.

La mobilisation des gouvernements municipaux dans la guerre prit la même orientation que celle adoptée par les zemstvos avec toutefois de fortes spécificités. L'Union des villes d'aide aux soldats malades et blessés (Vserossijskij sojuz

22. Ibid.,p. 162. Ce qui s'est traduit par l'exigence de restitution des sommes restantes allouées par l'État pour la campagne contre la famine de 1907.

23. D'après le plan de lutte contre la famine mis en place par les autorités, les zemstvos ne pouvaient plus agir que dans leur propre province. Quant aux provinces, dépourvues de zemstvos, c'était à la bureaucratie locale ainsi qu'aux maréchaux de la noblesse d'assurer la coordination de l'action. Lindenmeyr, Poverty is not a Vice ..., p. 193.

24. Polner, Žiznennyj put'́..., p. 163.

25. William Gleason, «The All-Russian Union of Zemstvos and World War One », in Emmons et Vucinich, eds., The Zemstvo in Russia... 
gorodov pomošči bol'nym i ranenym voinam) ${ }^{26}$ fut instaurée le 8 août 1914 par les maires de 46 municipalités, réunis en congrès à la Douma de Moscou. Comme pour les zemstvos, cette association constituait l'aboutissement d'un mouvement fédératif qui avait mobilisé les milieux réformateurs des doumas à partir de 1905. Mais, jusqu'à la guerre, la composante humanitaire de ce mouvement était restée en arrière-plan. Comme le souligne l'auteur d'une thèse sur l'Union des villes, les dimensions humanitaires et politiques de cette nouvelle organisation furent d'emblée indissociables. Nikolaj Astrov, le chef de file du courant progressiste de la Douma de Moscou et l'un des principaux fondateurs de l'Union, disait ainsi que la future réforme des municipalités dépendrait de l'intensité de la mobilisation des municipalités dans l'effort de guerre 27.

Le fait que les présidents des directoires des zemstvos, majoritairement conservateurs, aient choisi comme délégué-général de l'Union des zemstvos une personnalité comme le prince L'vov, libéral modéré ne pouvant se prévaloir d'aucune légitimité élective au sein du zemstvo, doit être souligné. C'est l'acteur aguerri de l'humanitaire qui s'imposa. Celui qui, pour faire avancer cette cause, avait fait preuve d'un grand pragmatisme en constituant un tissu relationnel dense des plus divers, englobant élus et professionnels des zemstvos et représentants des autorités impériales (y compris l'empereur) et du milieu associatif privé. Au sein de l'Union des villes également, le choix du délégué général, montre que l'expérience de l'action humanitaire comptait tout autant que la dotation d'un mandat électif. Mihail V. Čelnokov avait, en effet, activement participé aux actions de l'Organisation générale des zemstvos, ce qui lui avait valu d'être commis d'office à l'élaboration du plan d'évacuation des soldats blessés de l'Union des villes ${ }^{28}$. Ainsi, au début de la guerre, pour les organes directeurs des zemstvos et des municipalités, le passé en matière d'action humanitaire constituait un mode de légitimation fondamental pour assumer la direction des Unions.

\section{La montée en puissance des Unions}

En investissant le vaste chantier humanitaire ouvert par la guerre, les Unions des zemstvos et des villes se trouvèrent confrontées à une situation radicalement nouvelle, induite par les faiblesses de l'État dans la gestion sanitaire de l'armée et beaucoup plus largement dans la prise en charge de l'effort de guerre. Au début du mois de septembre 1914, le champ d'action des Unions avait été strictement délimité par les autorités militaires. Ni le front, ni les régions mêmes qui l'entouraient n'étaient accessibles aux Unions. Celles-ci ne pouvaient agir qu'à l'est d'une ligne de démarcation reliant Moscou à Kharkov, excluant ainsi de toute activité 15 zemstvos de province. Rapidement dépassé par l'afflux des blessés des campagnes

26. Gronsky, Astrov, The War and the Russian Government, p. 183.

27. A. Astašev, « Vserossijskij sojuz gorodov », Thèse, RGGU, Moscou, 1994, p. 46.

28. F. A. Seleznev, « Mihail Vasil’evič Čelnokov », Voprosy istorii, 6, 2004, p. 86 et 91. 
d'août et de septembre, le pouvoir militaire dut faire appel aux deux Unions pour qu'elles participent à l'évacuation des blessés dans les zones mêmes de combat. Les premiers soins d'urgence apportés aux blessés par des équipes médico-sanitaires (vračebno-sanitarnye otrjady), les évacuations en train sanitaire hors des zones de combat relèvent des aspects les plus importants du travail des Unions jusqu'en 1917. Ainsi, au 1er janvier 1917, les trains de l'Union des zemstvos avaient convoyé 1626531 soldats et ceux de l'Union des villes, $340000^{29}$.

Dès l'automne 1914, l'aide sanitaire à l'armée ne constitua plus qu'une des formes d'une participation plus vaste à l'effort de guerre. À la demande de l'armée, confrontée à un manque de matériel en tous genres, les Unions s'orientèrent désormais aussi vers son soutien logistique. L'approvisionnement de l'armée en vêtements d'hiver, chaussures, tentes, constitua une première étape, bientôt relayée par un engagement, à partir de l'été 1915, dans la production de matériel d'armement. À cet effet, les Unions mirent en place, en juillet 1915, un organe commun, le Comité directeur des unions des zemstvos et des villes pour l'approvisionnement de l'armée (Glavnyj komitet zemskogo i gorodskogo sojuzov po snabženiju armii) appelé Zemgor. Outre la production malgré tout modeste de munitions, le Zemgor participa au démantèlement et à l'évacuation des usines se trouvant dans les régions occidentales de l'empire menacées par l'avancée de l'ennemi, ainsi qu'à l'aménagement des nouvelles infrastructures (tranchées, routes, fortifications) provoqué par le déplacement du front de 500 km à l'est, consécutif à la « Grande Retraite » de l'été $1915^{30}$. Fin 1915, près de 100000 ouvriers et ingénieurs travaillaient sous les ordres du Zemgor ${ }^{31}$.

La mobilisation, par le biais du Zemgor, des Unions dans l'industrie de guerre s'inscrivait dans une volonté plus générale de participation des administrateurs et spécialistes des organes d'auto-gestion à l'élaboration de la politique économique de guerre. En effet, les nécessités de la guerre fournirent l'occasion aux élites sociales d'engager une collaboration avec les administrateurs gouvernementaux. Ces élites envisageaient leur partenariat avec l'État dans une perspective de renforcement de l'intervention étatique dans la restructuration de l'économie et de mobilisation générale de la société pour la guerre ${ }^{32}$, avec pour points cruciaux, l'approvisionnement des populations civiles et militaires en denrées alimentaires et la lutte contre la hausse des prix. L'intervention de spécialistes de l'agronomie venus des Unions dans la gestion de l'économie fut concrétisée par l'entrée de leurs représentants au Conseil spécial de l'approvisionnement, une instance gouvernementale instaurée le

29. T. I. Polner, Russian Local Government during the War and the Union of Zemstvos, New Haven, 1930, p. 90 ; Gronsky, Astrov, The War and the Russian Government, p. 202 ; Rossijskij Gosudarstvennyj Voenno-Istoričeskij Arhiv (RGVIA), f. 12564 (Glavnyj Komitet VZS), op. 1, d. 1187, 1. 50.

30. Le Zemgor a pris en charge l'évacuation de 150 usines du grand centre industriel de Riga. Polner, Russian Local Governement..., p. 278.

31. Ibid., p. 283.

32. Peter Holquist, « La société contre l'État, la société conduisant l'État : la société cultivée et le pouvoir d'État en Russie, 1914-1921 », Le Mouvement social, juillet-septembre 2001, p. 26. 
17 août 1915 et réunissant, sous la présidence du ministre de l'Agriculture, des représentants du ministère des Transports, de la Douma, du Conseil d'État, des Unions ainsi que des experts de l'économie. L'échec de cette brève collaboration était prévisible, notamment en raison d'une divergence de vues entre bureaucrates et spécialistes sur le sens à attribuer au dirigisme économique ${ }^{33}$. Toutefois, pour des raisons de conflits interministériels, c'est aux bureaux statistiques des zemstvos et non au Comité central de la statistique (Central'nyj statističeskij komitet) rattaché au ministère de l'Intérieur, que le ministère de l'Agriculture confia l'organisation du recensement agricole de l'été $1916^{34}$. Fonctionnant comme un organe de coordination des bureaux statistiques des zemstvos, le bureau statistique auprès du Département d'économie du Comité directeur de l'Union des zemstvos réalisait une aspiration ancienne des statisticiens du zemstvo, à savoir l'unification du travail statistique mené à l'échelle locale ${ }^{35}$. Comme l'a montré Martine Mespoulet, c'est dans le sillage de l'Union des zemstvos et non de l'État que se forgea l'expérience d'une administration statistique servant les besoins de l'économie de guerre.

La grande diversification des compétences des Unions entraîna, bien entendu, une mobilisation des ressources humaines, administratives et matérielles sans précédent. Au début de 1917, dans les seules zones de front, 170000 personnes travaillaient pour l'Union des zemstvos et 59000 pour l'Union des villes ${ }^{36}$. Les Unions durent faire face à des dépenses en constante augmentation, couvertes en grande partie par des fonds d'État ${ }^{37}$. À la fin de 1916, 7728 institutions - hôpitaux, trains sanitaires, cantines, dépôts, usines, etc, - étaient rattachées à l'Union des zemstvos. Leur encadrement impliquait désormais une lourde machine administrative qui se complexifiait, notamment en raison de la présence des Unions dans les zones de front et à l'intérieur de l'empire ${ }^{38}$.

33. La nécessité d'une économie dirigée était communément admise par les administrateurs et les économistes du Soveščanie, notamment pour ce qui concerne le contrôle des prix. En revanche, l'idée de réquisition des denrées, défendue par Petr Struve (Union des zemstvos) et Vladimir Groman (Union des villes) rencontra une forte opposition au sein de la bureaucratie. Alessandro Stanziani, « Spécialistes, bureaucrates et paysans. Les approvisionnements agricoles pendant la Première Guerre mondiale (1914-1917) », Cahiers du Monde russe, 36 (1-2), 1995, p. 76-77.

34. Outre l'appel à des statisticiens professionnels (ce qui n'était pas le cas de la majorité des statisticiens du CSK), il faut voir dans cette décision l'expression du conflit qui oppose alors les ministères de l'Intérieur et de l'Agriculture, Stanziani, ibid., p. 78.

35. Martine Mespoulet, Statistique et révolution en Russie. Un compromis impossible (18801930), Presses Universitaires de Rennes, 2001, p. 110-113.

36. N. Golovin, Voennye usilija v mirovoj vojne, t. 1, P., 1939, p. 188.

37. D'août 1914 à l'automne 1917, les Unions des zemstvos et des villes reçurent, à elles deux, près de 1,240 milliard de roubles. Astašev, Vserossijskij sojuz gorodov, p. 229 et 312.

38. Polner, Russian Local Governement..., p. 70. Outre la multiplication, au niveau des Comités directeurs, des sections chargées de coordonner les activités des Unions (pour le Comité directeur de l'Union des villes, 4 sections en 1914 et 21 sections en 1916), la grande nouveauté dans l'organigramme des Unions résidait dans la mise en place, à partir de novembre 1914, de structures administratives spécifiques aux zones militaires. Rattachés aux différents fronts, les Comités de front coordonnaient le travail effectué par les institutions des zemstvos et les municipalités dans ces territoires. 
Les services fournis à l'armée par les Unions, en matière de soins et d'évacuation des soldats blessés, posèrent les jalons de ce que l'on pourrait qualifier de discours de «l'efficacité », qui sous-tendait en permanence l'aspiration des Unions à occuper les espaces laissés vacants par l'État central. Comme l'affirmait le délégué général de l'Union des zemstvos, le prince L'vov, lors de son intervention inaugurale au congrès des délégués de l’Union, le 12 mars 1915 :

Ce n'est pas mentir que d'affirmer que toute la question de l'évacuation se trouve entre les mains des Unions des zemstvos et des villes [...]. Il est important de souligner, ici, que la rapidité avec laquelle les organisations publiques (obščestvennye) accomplissent leur travail est tout à fait inimaginable pour les services de l'État ${ }^{39}$.

Les liens privilégiés établis, dès l'automne 1914, par les délégués généraux et les responsables des Comités de front des Unions avec plusieurs hauts responsables de l'armée jouèrent un rôle essentiel dans cette représentation. En appelant les Unions à déployer leur action sanitaire au sein même des zones de front et en contribuant de façon substantielle à leur financement, le haut commandement militaire joua un rôle déterminant dans le changement d'échelle de l'action humanitaire des Unions. Cette présence des Unions dans des territoires entièrement placés sous juridiction militaire n'était pas neutre ${ }^{40}$. En effet, la restriction dans les zones militaires du champ de compétences des autorités civiles ne faisait que renforcer l'impression que, sur de larges fractions de territoire, l'État était absent de la prise en charge sanitaire des soldats blessés. Les notes prises par A. N. Jahontov au Conseil des ministres sont éloquentes sur la façon dont on percevait la situation :

Dans les zones militaires, on refuse l'accès aux services de l'État mais on réclame les Unions ${ }^{41}$.

Ou encore,

Danilov est à la limite de la folie, il a donné l'ordre à l'arrière, qui se trouve sous mes ordres [c'est le ministre de la Guerre Polivanov qui parle - O.P.-B.], de former un détachement de 80000 ouvriers. Et en plus, c'est l'Union des zems-

39. «Protokoly sobranija upolnomočennyh zemstv 12-13 mars 1915 », Izvestija Glavnogo Komiteta VZS, n¹2-13, 1915.

40. Conformément au règlement (Položenie o polevom upravlejnii vojsk v voennoe vremia) adopté par Nicolas II en juillet 1914, les territoires situés à l'ouest d'une ligne reliant la Finlande à la mer Noire (et comprenant la capitale) furent placés sous juridiction militaire. Avec la retraite de l'armée russe en été 1915 , cette juridiction se déplaça encore à $500 \mathrm{~km}$ à l'est. Les pouvoirs dont disposaient les autorités militaires s'appliquaient à toutes les sphères de la vie des populations locales. Daniel Graf, « Military Rule Behind the Russian Front, 1914 1917 : The Political Ramifications », Jahrbücher für Geschichte Osteuropas, 22, 1974, p. 393.

41. Séance du Conseil des ministres du 18 septembre 1914. Sovet Ministrov Rossijskoj imperii v gody Pervoj mirovoj vojny. Bumagi A. N. Jahontova (zapisi zasedanij i perepiska), SPb., 1999, p. 70. 
tvos qui en est chargée et qui nous réclame maintenant près de 300 automobiles $^{42}$.

Dans un contexte de tension permanente entre autorités civiles et militaires concernant le partage des compétences dans ces zones, l'état-major instrumentalisa l'action des Unions en soulignant leur efficacité face à l'inaction du pouvoir civil. Une enquête, menée à l'instigation du chef du ravitaillement du front ouest, le général N. A. Danilov, auprès de plusieurs chefs d'armée sur leur appréciation du travail accompli par les Unions dans leurs zones respectives, le confirme parfaitement ${ }^{43}$. D'après la conclusion de l'enquête établie par Danilov lui-même, l'Union des zemstvos et, dans une moindre mesure, l'Union des villes surent se rendre indispensables au front. Le contraste est ici établi avec l'incompétence manifeste, toujours selon Danilov, des initiatives émanant des autorités civiles.

De leur côté, dans un souci de consolidation de leur position face aux autorités civiles, les Unions n'hésitèrent pas à rendre publiques et à diffuser les appréciations positives des hauts responsables militaires sur leurs actions auprès de l'armée ${ }^{44}$. Ces déclarations participaient à la mise en place d'une image de partenariat exclusif entre les organisations sociales et l'armée. Ce partenariat avait d'autant plus d'importance qu'il constituait le principal garant de l'existence des Unions. Le sort de celles-ci restait toujours aléatoire en raison du caractère ambigu de leur statut et des attaques du gouvernement dont elles firent l'objet à partir de $1916^{45}$. Ainsi, à propos des relations de l'Union avec l'armée, le représentant du comité du front sud-ouest de l'Union des zemstvos, E. A. Elačič, soulignait :

L'Union des zemstvos a grandi, on s'est habitué à elle, on tient compte de ses avis, on a recours à elle. Devenue partie intégrante de l'armée, il serait impossible de l'en arracher ${ }^{46}$.

Comme le soutient Alexandre Astašev, les succès rencontrés par les Unions dans le domaine humanitaire furent déterminants pour la prise de conscience, par les responsables des Unions, des capacités de l'obščestvo à résoudre tous les

42. Séance du Conseil des ministres du 13 septembre 1915, ibid., p. 271. Le général N. A. Danilov, en tant que chef du ravitaillement des armées du front ouest, était doté de pouvoirs pouvant être qualifiés de dictatoriaux sur les zones situées à l'arrière du front. Graf, « Military Rule Behind the Russian Front... », p. 392.

43. Cette enquête sur les Unions manifeste ainsi son intérêt pour des organismes dont il cherche à s'assurer la plus grande loyauté. RGVIA, f. 2049, op. 1, d. 447, 1. 29-46.

44. Voir, à titre d'exemple, les télégrammes du général Danilov et du général Litvinov (chef de la $1^{\text {re }}$ armée) in « Sobranie upolnomočennyh gubernskih zemstv 12-13 marta 1915 », Izvestija GK VZS, n 12-13. Priloženie, 1915, p. 15.

45. Les Unions avaient été reconnues par décret impérial en septembre 1914 « pour la durée de la guerre », mais leur légalisation ne survint que sous le Gouvernement Provisoire. Gronsky, Astrov, The War and the Russian Government, p. 185. A. S. Tumanova, Samoderžavie i obščestvennye organizacija v Rossii, 1905-1907, Tambov, 2002, p. 381-382.

46. Discours prononcé au congrès des délégués du VZS le 12 mars 1916. Sobranie upolnomočennyh gubernskih zemstv 12-14 marta 1916, Moscou, 1916, p. 74. 
problèmes relevant de l'État, notamment ceux relatifs aux questions économiques et sociales liées à la guerre ${ }^{47}$. On ajoutera qu'au fur et à mesure de l'installation de la Russie dans le conflit, les opérations d'assistance conduites par les Unions furent, elles aussi, pénétrées par l'esprit de planification et de rationalisation. À partir de 1915, le terme de "planomernost'» (planification, organisation) était devenu omniprésent dans le vocabulaire des animateurs des Unions. Ces derniers y voyaient l'une des manifestations de la capacité des Unions à assumer des tâches étatiques. L'idée de planomernost's'imposa ainsi dans tous les domaines d'activité et, au premier chef, dans celui de l'action humanitaire. Dans son discours prononcé au congrès de l'Union des zemstvos du 12 mars 1916, E. A. Elačič caractérisait les différentes phases de l'expansion de l'Union. Il montrait ainsi comment l'Union des zemstvos avait tout d'abord agi au coup par coup, au gré des besoins et des demandes. Désormais, disait-il, «l'Union des zemstvos doit procéder de façon planifiée et systématique (systematizirannaja) et suivre les priorités qu'elle aura elle-même définies ${ }^{48}$.

\section{Les Unions et les réfugiés : d'une pratique d'assistance vers l'ambition d'une politique de gestion des transferts de populations}

L'action entreprise par les deux Unions en faveur des populations civiles déplacées, au cours du printemps et de l'été 1915 , est emblématique de cette volonté de rationalisation des pratiques d'assistance. Elle mérite, à ce titre, que l'on s'y attarde.

La pénétration des armées allemande et austro-hongroise en territoire russe et la retraite des troupes russes à $500 \mathrm{~km}$ de leur position initiale furent à l'origine d'un vaste mouvement d'évacuation des populations civiles des zones de front ${ }^{49}$. Ce mouvement résultait moins de la fuite spontanée des populations locales que d'une politique d'expulsion massive (pogolovnoe vyselenie), menée par le haut commandement militaire ${ }^{50}$. Les mesures d'expulsion adoptées par les autorités militaires à l'encontre des populations civiles s'inscrivaient en partie dans une tactique de terre brûlée, à savoir la destruction systématique des villages abandonnés à l'ennemi ${ }^{51}$.

47. Astašev, Vserossijskij sojuz gorodov, p. 229.

48. Sobranie upolnomočennyh... 1916, p. 74-75.

49. En s'appuyant sur différents comptes tenus au cours de la guerre, A. N. Kurcev estime le nombre total de réfugiés à cinq millions. Il s'agit d'une évaluation inférieure à celle du démographe soviétique, E. Volkov, qui compte lui près de dix millions de réfugiés pour toute la période de la guerre. E. Z. Volkov, Dinamika narodonaselenija SSSr za 80 let, Moscou, 1930, p. 68-75 ; A. N. Kurcev, « Bežency pervoj mirovoj vojny (1914-1917)», Voprosy istorii, 8, 1999.

50. Peter Gatrell, A Whole Empire Walking, Refugees in Russia during World War I, Bloomington : Indiana University Press, 1999.

51. Les pratiques de «nettoyage global » des territoires (pogolovnoe očiščenie) furent surtout le fait de plusieurs chefs d'armée (notamment de la $3 \mathrm{e}$ et de la 10 ) ainsi que des commandants agissant au niveau des corps d'armée. Au cours d'une réunion du 23 juin 1915, consacrée aux « modes de nettoyage de certains territoires par les troupes », l'état-major opta pour l'abandon 
Elles suivaient aussi une logique de stigmatisation de certaines minorités nationales, particulièrement nombreuses dans les régions occidentales de l'empire placées sous le contrôle de l'armée ${ }^{52}$. Les Ukrainiens de Galicie orientale, les Polonais et surtout les Allemands et les Juifs constituèrent les cibles privilégiées de ces mesures.

Par l'inquiétude qu'elle suscita auprès des responsables politiques et « sociaux », la migration forcée des populations vers l'intérieur de l'empire contribua à alourdir un climat politique déjà sous tension ${ }^{53}$. En effet, le problème des réfugiés constitua un facteur de déstabilisation supplémentaire des relations entre autorités militaires et civiles, ces dernières critiquant violemment l'armée pour sa gestion désastreuse de l'évacuation des populations. Il participa également au renforcement de l'antagonisme, manifeste depuis la crise des munitions de l'hiver 1914-1915, entre autorités civiles d'une part et parlementaires et acteurs «publics » de l'autre. Comme nous le savons, cet antagonisme aboutit, en juillet 1915, à la constitution, au sein de la Douma, du Bloc progressiste (Progressivnyj blok), dont la principale revendication était la formation d'un nouveau "gouvernement bénéficiant de la confiance du pays » (pravitel'stvo pol'zjujuščeesja doveriem strany) ${ }^{54}$. Quant aux organes directeurs des Unions, la « delovaja pozicija », ou l'idée que la mobilisation pour l'effort de guerre devait primer sur l'action politique, fut relativisée, y compris chez ses principaux défenseurs, L'vov et Čelnokov, avec le renvoi de la Douma le 3 septembre ${ }^{55}$. Aux yeux des libéraux, K.-D. notamment, les congrès des Unions, prévus du 7 au 9 septembre, étaient appelés désormais à exercer un rôle de tribune pour l'opposition libérale ${ }^{56}$. Et surtout, à la demande des membres du Bloc progressiste de la Douma, il revenait désormais aux Unions de former une députation, afin

de ces pratiques. Toutefois, la Stavka revint sur ses positions et se prononça, à diverses reprises, pour l'expulsion de tous les habitants de certains secteurs des provinces de Kovno et de Grodno. Rapport sur la réunion de la Stavka du 23 juin 1915. RGVIA, f. 2005, d. 42 (1), 1. 8. A. N. Kurcev, « Beženctvo », in N. N. Smirnov, éd., Rossija i Pervaja mirovaja vojna, SPb. : D. Bulanin, 1999, p. 132.

52. Eric Lohr, Nationalizing the Russian Empire : the Campaign against Enemy Aliens during World War I, Cambridge, MA : Harvard University Press, 2003.

53. Les notes prises par A. Jahontov lors des séances du Conseil des Ministres de l'été 1915 constituent l'un des meilleurs témoignages sur la crise de l'été 1915. Jahontov, Sovet ministrov Rossijskoj imperii..., p. 178-282.

54. V. S. Diakin, Russkaja buržuazija i tsarim v gody pervoj mirovoj vojny, L., 1967, p. 96109.

55. Comme le montre A. Astašev, la « delovaja pozicija » des membres du Comité directeur de l'Union des villes était dictée à la fois par le refus d'exercer un chantage sur l'État en temps de guerre, mais aussi par le fait que les Unions se trouvaient dans une phase de coopération avec les autorités gouvernementales à travers leur participation aux différents Conseils spéciaux mis en place par le Gouvernement en août 1915. Astašev, Vserossijskij sojuz gorodov, p. 69.

56. Au cours de la réunion du Comité central du parti K.-D. du 5 septembre, D. I. Šahovskoj s'exprime dans les termes suivants: "Toute l'attention doit être portée désormais sur les Unions des zemstvos et des villes. Il n'y a pas de Douma, mais il y a les congrès des Unions [...]. Leur voix sera la voix de tout le pays ». Protokoly Central'nogo komiteta konstitucionnodemokratičeskoj partii, 1915-1920, M. : ROSSPEN, 1998, p. 162. 
de remettre au tsar, en mains propres, un mémorandum sur la crise politique traversée par le pays ${ }^{57}$.

C'est dans ce contexte où les Unions, en pleine expansion, occupaient le devant de la scène politique que se mit en place la mobilisation en faveur des populations civiles déplacées.

À l'instar des acteurs étatiques et de la société civile, les Unions furent tout d'abord surprises par le caractère inattendu et brutal du problème des réfugiés, il apparaît néanmoins que leur action fut d'emblée pensée en termes de « mise en ordre à partir du chaos $»^{58}$. Nous ne pouvons que suivre les conclusions de Peter Gatrell, selon lesquelles les élites sociales étaient profondément marquées par l'image du réfugié comme vecteur de désordre ${ }^{59}$. Les propos du président du comité du front sud-ouest de l'Union des zemstvos, Šlikevič, sont parfaitement clairs sur ce sujet :

Cette horde de nomades démoralisés, indisciplinés s'avance en Russie. Elle la menace d'un terrible fléau. Elle la conduit à la ruine, au pillage et à la désorganisation complète du pays ${ }^{60}$.

Ainsi dans le cas des réfugiés, la planomernost' qui sous-tendait tout le projet humanitaire des Unions participait aussi de l'idée de préservation d'un ordre social menacé par l'arrivée d'éléments supposés incontrôlés dans les régions intérieures de l'empire.

L'évacuation des populations civiles durant la Grande Retraite amène à s'interroger sur le rôle joué par les Unions des zemstvos et des villes. On assiste, en effet, à la transformation d'une action d'assistance aux populations déplacées en une tentative d'encadrement de ces populations, mais aussi d'organisation de leur évacuation des zones de front vers l'intérieur de l'empire. Ceci signifiait donc que les Unions se considéraient, certes comme des relais, mais aussi comme des parties intégrantes de la chaîne de décision de l'évacuation, au même titre que les autorités miltaires et civiles.

La mobilisation des Unions des zemstvos et des villes auprès des populations déplacées débuta dans la seconde quinzaine de juin, alors que les déplacements massifs de populations avaient été enclenchés depuis la fin du mois d'avril. Les réfugiés et expulsés provenaient essentiellement de Galicie, Volhynie et Podolie, $\mathrm{du}$ royaume de Pologne et des régions baltes. Comme le rappelait l'auteur d'un important rapport sur les réfugiés, publié par l'Union des villes en 1915, les « réfugiés ont inondé les postes de ravitaillement situés à proximité de l'armée, puis ils se sont précipités vers l'arrière immédiat de l'armée en retraite ${ }^{61}$. Cette mobili-

57. La députation dirigée par le prince L'vov devait être composée de 6 personnes, soit 3 représentants par Union. Le refus de Nicolas II de recevoir la députation marque une étape importante dans la rupture de confiance entre L'vov et la monarchie.

58. Gatrell, A Whole Empire Walking...,p. 80.

59. Ibid., p. 79.

60. Lettre de Šlikevič, reçue par le Comité directeur du VZS, le 14 août 1915. RGVIA, f. 13273 , op. 1, d. 104, p. 5 .

61. Bežency i vyselency, M., 1915, p. 5. 
sation relève moins d'une initiative interne aux Unions que d'une impulsion venant de l'extérieur à savoir de l'armée, qui cherchait à remettre à des interlocuteurs non militaires, mais pas nécessairement issus de la bureaucratie civile, la responsabilité de la prise en charge des populations des territoires qu'ils évacuaient. Sollicitées par l'armée, mais aussi par les administrations locales, les organisations publiques furent donc investies d'une nouvelle tâche qui témoignait, une fois de plus, de leur solide implantation sur le front ${ }^{62}$. Comme le soulignait l'auteur du rapport, en raison de l'extrême confusion qui régnait dans les zones d'évacuation, les premières actions des Unions furent placées sous le signe de la plus grande improvisation, sans qu'il y ait de coordination des opérations par les comités directeurs ${ }^{63}$. Nikolaj Petuhov, envoyé en mission en juillet dans la région de Vlodava (province de Grodno) par le comité directeur de l'Union des zemstvos, témoigne également du manque d'informations sur les initiatives des uns et des autres et dont souffraient, au début, les responsables locaux des Unions.

Le problème le plus aigu que connaisse cette région, c'est les réfugiés et c'est justement ici que les Unions doivent aider. Il paraît que des initiatives ont déjà été prises. [...] Organiser cette action est très difficile, car il n'y a rien de plus anarchique que ce flot de réfugiés ${ }^{64}$.

Les témoignages des responsables de l'Union des zemstvos se trouvant sur place concordent pour dire que le ravitaillement systématique des populations était impossible en raison du caractère chaotique de leur évacuation. Dans un premier bilan établi à la mi-août, $\mathrm{N}$. Hmelev, membre du Comité directeur de l'Union des zemstvos et présent depuis le début de juillet sur les lieux d'évacuation du front sud-ouest, qualifie l'action d'assistance de l'Union des zemstvos envers les réfugiés de « très ponctuelle » (slučajnaja).

Nous avons mis en place, sur les lieux de passage des réfugiés, un certain nombre de postes de ravitaillement mais nous ne pouvons, en aucun cas, dire que nous travaillons de façon systématique. Or, il est absolument indispensable de procéder à une véritable organisation et planification de cette action.

Pour illustrer ces propos, Hmelev évoque les difficultés rencontrées par le personnel des postes de ravitaillement pour localiser les réfugiés. Le problème était

62. Sur le front sud-ouest, le haut commandement appela les Unions des zemstvos et des villes, à partir de la mi-juin, à participer à l'organisation du ravitaillement et de la prise en charge des réfugiés de Galicie évacués vers les provinces de Volhynie et de Podolie. Sur le front nordouest, c'est le 21 juin que le général Danilov s'est adressé aux Unions, leur demandant d'organiser l'aide alimentaire et sanitaire sur l'itinéraire des évacués des provinces de Holm, de Lublin et de Grodno. RGVIA, rapport de Danilov sur la question réfugiée, f. 2018, d. 48 (2). Lettre du chef d'intendance adjoint du front sud-ouest, le général El'sner au prince L'vov, le 23 juin 1915, RGVIA, f. 12564, op. 1, d. 54,1.7.

63. Bežency $i$ vyselency, p. 9.

64. N. G. Petuhov, Brest-Litovsk, 19 juillet 1915. Je tiens à remercier M. Alexis Rastorguev d'avoir mis à ma disposition les lettres du front de N. Petuhov. 
particulièrement crucial le long des voies de chemins de fer et dans les gares en particulier. Il évoque ainsi l'épisode d'une équipe médico-sanitaire de l'Union chargée d'assurer le ravitaillement des réfugiés arrivant en gare de Kiev, qui, pendant près d'une semaine, avait parcouru en vain toutes les gares de la région sans jamais réussir à trouver ces fameux trains ${ }^{65}$.

Plusieurs mesures furent adoptées localement pour mieux connaître et suivre le mouvement des réfugiés. Le Comité du front sud-ouest de l'Union des zemstvos, situé en première ligne des évacuations, mit ainsi en place un système de liaisons télégraphiques avec les zemstvos et les organisations caritatives locaux. Il envoya également des agents enquêter dans les différentes provinces du sud-ouest et, mesure originale, il fit appel à des guides-accompagnateurs (le plus souvent des prêtres et des étudiants) chargés d'accompagner les réfugiés dans les trains jusqu'à leur lieu de destination et de se poser en interlocuteurs des administrations locales et ferroviaires ${ }^{66}$. Cette dernière initiative s'avéra par la suite l'une des mesuresphares de l'action d'assistance des Unions envers les populations civiles déplacées. Entre septembre et décembre 1915, ces guides escortèrent un millier de trains, convoyant près de un million de réfugiés ${ }^{67}$.

Le pessimisme manifesté par certains responsables des Unions en prise directe avec les difficultés posées par les évacuations et le caractère improvisé des premières actions ne doivent donc pas masquer l'importance de ce qui fut entrepris au cours des premières semaines. L'Union des zemstvos ouvrit ainsi près d'une centaine de postes de ravitaillement sur le front nord-ouest et environ soixante-dix sur le front sud-ouest ${ }^{68}$.

La mobilisation des Unions à plus grande échelle débuta à la mi-juillet. C'est à ce moment que les zemstvos et surtout les municipalités des provinces, tout d'abord de la périphérie puis de l'intérieur de l'empire, commencèrent à adresser en masse aux Comités directeurs des demandes de subsides pour leurs actions de prise en charge des réfugiés. Dans la seconde quinzaine de juillet, les deux capitales, naturellement, mais aussi des villes du Povolž'e et de l'Oural étaient devenues des grands lieux de concentration pour les populations déplacées. Le Comité directeur de l'Union des villes, en la personne de N. N. Ščepkin, lança alors une vaste enquête auprès des municipalités afin d'évaluer à la fois l'ampleur du phénomène et les moyens requis pour y répondre. ${ }^{69}$ Dans un premier temps, l'idée de planification des actions d'assistance des Unions suivit deux orientations : d'une part, celle de l'organisation des mouvements d'évacuation et, de l'autre, l'évaluation des

65. N. Hmelev à G. E. L'vov. Lettre reçue le 14 août 1915. RGVIA, f. 13273, op. 1, d. 104, 1. 2. Les postes de ravitaillement du comité du front sud-ouest distribuèrent presque 10 millions de repas entre juin et décembre 1915. Polner, Russian Local Governement..., p. 163.

66. « Obščij obzor dejatel'nosti otdela pomošči bežencam pri komitete Jugo-zapadnogo fronta VZS », Izvestija GK VZS, 26, 1 ${ }^{\text {er }}$ novembre 1915, p. 91.

67. Gronsky, Astrov, The War and the Russian Government..., p. 236.

68. Ivestija VSG VZS, 22-23, 15 septembre 1915. Izvestija VSG GK, 24, 1 1 $\mathrm{er}$ octobre 1915.

69. Bežency $i$ vyselency, p. 9. 
besoins financiers des organes locaux et de la coordination de la répartition des subsides.

En outre, cette aspiration à apporter des solutions planifiées (planomernye) au problème de l'évacuation et de la prise en charge des déplacés conduisit les responsables des Unions à envisager de coordonner leurs initiatives et de définir un programme d'action commun. Cette initiative aboutit le 22 août 1915 à la formation d'un comité commun, la Section d'aide aux réfugiés des Unions des zemstvos et des villes (Otdel po ustrojstvu bežencev vserossijskogo i vsegorodskogo sojuzov) appelé communément Sobež.

Un plan d'évacuation fut établi le 18 juillet à Brest-Litovsk, au cours d'une grande conférence commune aux deux Unions. Divisé en trois axes d'évacuation (direction nord-est, centre et sud-est), ce plan, dont l'auteur principal, S. N. Bahrušin, était membre du Comité directeur de l'Union des villes, avait pour orginalité d'établir plusieurs niveaux d'intervention des Unions, en fonction des étapes. Sur la première ligne d'évacuation, dans les zones d'opérations militaires, l'aide devait être strictement alimentaire, en sorte de ne pas ralentir le rythme d'évacuation. La deuxième étape, située à l'arrière, consistait à assurer un contrôle sanitaire des arrivants, à procéder à un premier enregistrement statistique minimal, puis à orienter les réfugiés vers les centres d'évacuation de l'intérieur. Elle visait à protéger les grands centres urbains d'un afflux désorganisé de réfugiés. Enfin la troisième étape, située dans les villes dites de répartition (raspredeliteli) comme Petrograd, Moscou, Orel, Koursk, Kharkov et Ekaterinoslav, avait pour objectif de procéder à un enregistrement définitif des réfugiés et de les répartir en fonction de leur aptitude au travail (trudosposobnost $)^{70}$.

L'élaboration du plan d'évacuation ainsi que la question du financement des actions engagées en faveur des réfugiés donnèrent lieu, tout au long du mois d'août, à une intense activité des délégués généraux pour obtenir le soutien des instances militaires et gouvernementales. Le principe des étapes-filtres notamment trouva un écho positif auprès du général Danilov dont les services financèrent des étapes se trouvant dans sa zone de commandement (front nord-ouest). Avec l'accélération, à partir du début août, de la retraite de l'armée russe et donc du cours de l'évacuation des populations civiles, ce plan devint néanmoins obsolète. Le refus des autorités gouvernementales d'accepter les propositions des Unions mit définitivement un terme à leur tentative d'organiser l'évacuation des réfugiés vers l'intérieur de l'empire.

L'aspiration des organisations publiques à apporter une réponse alternative en l'absence de toute action de planification des évacuations par les autorités centrales trouva un obstacle majeur dans l'élaboration d'une politique gouvernementale visant à affirmer l'autorité de l'État dans la gestion de la question des réfugiés. Fin juillet, l'apparition dans les zones de front de deux « délégués plénipotentiaires au placement des réfugiés» (osoboupolnomočennye po ustrojstvu bežencev), nommés par le ministère de l'Intérieur pour superviser l'évacuation des populations 
civiles vers les régions intérieures de l'empire, constitua la première étape de la tentative d'éviction des Unions. Celles-ci ne tardèrent pas à réagir. Dans un mémorandum adressé en août au ministère de l'Intérieur, le délégué général de l'Union des villes, M. Čelnokov, dénonça l'incompétence des délégués plénipotentiaires dans l'évacuation des réfugiés qu'il disait « dépourvue de planification ». Il critiquait, en particulier, le fait que les réfugiés étaient envoyés dans des provinces sans enquête préalable sur les capacités d'accueil ${ }^{71}$.

La question du financement, déterminante pour la mise en œuvre d'une action d'assistance à grande échelle, constitua le second aspect du bras de fer qui opposait les Unions au gouvernement. Dès la fin de juillet, les délégués généraux des Unions multiplièrent les télégrammes au ministère de l'Intérieur, demandant le remboursement des frais déjà occasionnés par les actions engagées, ainsi que le financement des mesures préconisées dans le plan d'évacuation. Justifiant leur demande par les multiples requêtes d'intervention des autorités militaires et les appels des zemstvos et des municipalités, les délégués généraux connurent les plus grandes difficultés pour obtenir une réponse claire de la part du ministère de l'Intérieur. Dans une lettre du 13 août 1915 adressée à L'vov, le représentant du ministère de l'Intérieur chargé de la question des réfugiés fit état, en l'absence d'une loi (celle-ci était en cours de préparation), de l'impossibilité de satisfaire les demandes de crédits. Mais, le 18 août, il accepta finalement d'accorder un million de roubles aux deux Unions. Puis fin août, le ministre de l'Intérieur, A. N. Ščerbatov, précisa que les demandes de subsides devaient désormais être adressées directement aux délégués plénipotentiaires et, pour les plus urgentes, aux chefs de ravitaillement des fronts (načal'niki snabženija armij) ${ }^{72}$.

Un Conseil spécial aux réfugiés (Osoboe soveščanie po ustrojstvu bežencev) fut créé en septembre à l'initiative du ministère de l'Intérieur. Cet organe mixte réunissait des représentants de la bureaucratie, de la Douma, ainsi que des principales organisations caritatives (Croix-Rouge, Comité Tatiana, comités nationaux) et des Unions. Son but, qui était de superviser l'action gouvernementale envers les réfugiés, contribua encore à étouffer les aspirations des Unions à jouer un rôle directeur dans la politique générale envers les réfugiés et également à centraliser les actions menées au niveau local par les organes d'auto-administration. En effet, la loi du 30 août relative aux réfugiés octroyait aux zemstvos et aux municipalités la responsabilité de l'aide et de l'installation des réfugiés dans les provinces d'accueil73. Dans cette perspective, le 17 septembre, le prince L'vov présenta devant le Conseil spécial

71. « Dokladnaja zapiska VSG v MVD » (non datée), Bežency $i$ vyselency, p. 20.

72. Pour un récapitulatif de la correspondance entre l'Union des zemstvos et le ministère de l'Intérieur, voir M. M. Ščepkin, Bežency i organizacija pomošči im v svjazi s rabotami Osobogo Soveščanija, Moscou, 1916, chapitre 1. D’une façon générale, le prince Urusov, délégué pour le front sud-ouest, opta pour une politique de collaboration avec les organisations publiques. Le Comité du front sud-ouest de l'Union des zemstvos a reçu de la part d'Urusov des subsides s'élevant à 17 millions de roubles, rendant possible la mise en œuvre d'importants programmes pour les réfugiés. Rapport de Šlikevič à L'vov (non daté), RGVIA, f. 13273, d. 104, 1. 110.

73. Le fait que le rôle directeur dans l'organisation de l'accueil des réfugiés, au niveau local, ait été attribué non pas aux gouverneurs, mais aux zemstvos et aux municipalités, résulte d'un amendement voté sous l'influence des parlementaires K.-D. 
les conclusions adoptées par les délégués des Unions lors des congrès du 79 septembre : le rôle assigné par la loi du 30 août aux organes d'auto-administration dans la gestion de l'accueil des réfugiés justifiait pleinement que la coordination des actions soit assurée par les comités directeurs des Unions ${ }^{74}$. Toute la politique du ministère de l'Intérieur revint à ignorer les revendications maintes fois exprimées par les deux représentants des Unions au Conseil, le prince L'vov et M. M. Ščepkin et à contourner les Comités directeurs, en assignant le plus souvent possible les crédits directement aux zemstvos et aux municipalités. Ces pratiques eurent l'effet escompté dans la mesure où le 16 novembre 1915, le Comité directeur de l'Union des zemstvos renonça à demander lui-même les crédits pour les comités locaux et appela ces derniers à s'adresser directement au Conseil spécial aux réfugiés ${ }^{75}$.

Les autorités gouvernementales n'étaient pas les seules à vouloir éviter que les Unions ne prissent sur elles la coordination de l'aide aux réfugiés dans les provinces. Les séances du Conseil furent le cadre d'un débat parfois conflictuel entre les Unions et les représentants des autres organisations caritatives et nationales d'aide aux réfugiés. Ainsi, le 10 octobre 1915, lorsque la commission des crédits du Conseil aborda la question du remboursement des sommes dépensées par les Unions pour les réfugiés, tous ses membres (moins le représentant des Unions, M. M. Ščepkin) votèrent une résolution déclarant que la centralisation par les Unions des actions des zemstvos et des municipalités en faveur des réfugiés contrevenait à la loi du 30 août. Ščepkin expliquait ce vote des organismes nationaux d'assistance aux réfugiés par le fait que ceux-ci préféraient intervenir directement auprès du Conseil spécial aux réfugiés pour défendre les intérêts de leurs propres réfugiés nationaux plutôt que de passer par l'intermédiaire des Unions ${ }^{76}$. En ce sens, la revendication, par les Unions, de la coordination des activités menées par les municipalités et par les zemstvos au niveau local pouvait être perçue comme un obstacle à cette aspiration ${ }^{77}$.

Le président du Comité de la grande-duchesse Tatiana d'aide aux victimes de guerre, le sénateur A. B. Neidgardt était le principal promoteur du vote contre les Unions, plus encore que les représentants des organisations nationales d'assistance aux réfugiés. Cette organisation caritative issue de la cour impériale et des milieux de la haute bureaucratie était présente dans les zones de combat depuis le début de la guerre ${ }^{78}$. C'est en Pologne, puis en Galicie que le Comité Tatiana avait déployé

74. Izvestija GK VZS, 24, 1 ${ }^{\text {er }}$ octobre 1915, p. 85.

75. Izvestija GK VZS, 28, 1 ${ }^{\text {er }}$ décembre 1915, p. 67.

76. Ščepkin, Bežency i organizacija pomošči..., p. 32.

77. Ceci n'a pas empêché les organisations nationales de coopérer étroitement dans les provinces avec les comités locaux du Sobež.

78. Dirigé par le conseiller d'État A. B. Neidgardt, le comité directeur était constitué de membres de la haute bureaucratie impériale et de représentants de l'industrie et de la société civile (le prince L'vov en faisait partie). Le comité exécutif était également relayé par un important réseau de comités locaux répartis dans les principales provinces de la Russie d'Europe. Le Comité Tatiana était financé par les fonds d'état avec une participation, néanmoins, importante de fonds collectés. Komitet ee imperatorskogo Vysočestva velikoj knjažny Tatiany Nikolaevny po okazaniju vremennoj pomošči postradavščim ot voennyh dejstvij, 14 sentjabrja 1914 po janvarja 1916, Petrograd, vol.1, 1916. 
une intense activité en fournissant une aide matérielle (nourriture, vêtements, logements) aux populations locales ruinées par le conflit. Depuis le début de l'été 1915, le Comité Tatiana s'était engagé activement à son tour dans l'assistance aux populations civiles évacuées ${ }^{79}$. Le soutien de la bureaucratie locale, mais aussi l'énergie déployée par les sections locales du Comité pour mobiliser les sociétés civiles (y compris certains représentants des zemstvos et des municipalités) dans les provinces d'accueil des réfugiés et, enfin, la stature acquise par le Comité Tatiana à travers ses activités, faisaient que cette organisation pouvait légitimement prétendre prendre part activement aux orientations du Conseil spécial aux réfugiés.

C'est dans cette perspective qu'au cours de la séance du 10 octobre, A. B. Neidgardt proposa de «mettre les points sur les "i" en ce qui concerne le rôle des Unions dans l'assistance aux réfugiés ». Selon lui, la coordination par les Unions des activités menées par les organes d'auto-administration dans ce domaine réduirait à néant le travail du Conseil spécial aux réfugiés, dont le but était justement de superviser toutes les institutions et les organisations chargées des réfugiés ${ }^{80}$.

Ce conflit larvé entre le Comité Tatiana et les Unions exprime, de fait, un certain glissement de l'idée de collaboration traditionnelle entre acteurs publics et acteurs privés (qui restait néanmoins forte à l'échelle locale) vers une prise de distance respective. Aux yeux des responsables des Unions, le terme blagotvoritel'nyj (caritatif) était de plus en plus associé à celui de slučajnyj (épisodique, ponctuel) ${ }^{81}$. Ce qui revient à dire que la planomernost'était pour eux inhérente à l'action publique et échappait à la charité privée. De son côté, le Comité Tatiana, (c'était aussi le cas de certains comités nationaux) tendait aussi vers une rationalisation de ses méthodes de travail, comme en témoigne l'adoption du principe de coordination par un comité directeur des actions menées par des sections locales ${ }^{82}$. Cette double évolution explique, en partie, la violence du conflit qui éclata en novembre 1915 autour de l'enregistrement statistique des réfugiés, action majeure pour les deux organisations concurrentes.

Pour les Unions, cet enregistrement des données statistiques, tant dans les zones d'évacuation que dans les provinces d'accueil, était considéré comme une activité prioritaire. Il devait permettre de systématiser les opérations de recherche des personnes disparues et surtout d'organiser l'évacuation et la répartition spatiale des réfugiés ainsi que le travail des zemstvos et des municipalités dans les provinces d'accueil. La pratique de comptabilité des réfugiés menée dans un cadre humani-

79. Par exemple, à partir de la fin juin, le comité Tatiana mena une action particulièrement énergique dans les provinces de Vitebsk et de Pskov, provinces de transit pour les réfugiés en provenance des provinces baltes, en assumant presque à lui seul (en dehors des associations juives) le ravitaillement et les premiers soins médicaux aux réfugiés arrivant dans les villes de Dvinsk, Vitebsk et Pskov. Bežency $i$ vyselency, p. 60-75.

80. Ščepkin, Bežency i organizacija pomošči..., p. 30

81. « Dokladnaja zapiska VSG v MVD », août 1915, Bežency i vyselency, p. 20.

82. Pour les comités nationaux d'aide aux réfugiés, voir Gatrell, A Whole Empire Walking..., chapitre 7 ; S. Zipperstein, «The Politics of Relief : The Transformation of Russian Jewish Communal Life during World War », Studies in Contemporary Jewry, 4, 1988, p. 33. 
taire est donc sortie de ce cadre pour s'inscrire dans une logique d'analyse globale d'un phénomène migratoire sans précédent.

L'enregistrement des réfugiés résulta de la conjonction de deux initiatives. En amont, dans les régions proches du front, le personnel chargé du ravitaillement des réfugiés ainsi que les guides-accompagnateurs commencèrent à procéder à l'enregistrement des réfugiés le long des routes, ainsi que dans les villes de transit et dans les gares. Les informations journalières recueillies (provenance, destination, nombre de portions journalières distribuées) étaient ensuite centralisées par les comités de province, puis envoyées trois fois par mois au Bureau statistique du Sobež. D'après les données fournies par 54 postes de ravitaillement faisant office de bureaux d'enregistrement, près de 105000 réfugiés passèrent par les bureaux le 16 septembre, premier jour d'enregistrement, le maximum ayant été atteint le 5 octobre (205 000 réfugiés). Avec la fin des évacuations, le nombre des réfugiés enregistrés commença à décroître à partir de la seconde moitié d'octobre (13 000, le 5 novembre 1915$)^{83}$. En aval, lorsque les réfugiés commencèrent à pénétrer dans les régions intérieures de l'empire, les comités locaux des Unions mobilisèrent leurs statisticiens pour évaluer le nombre d'arrivants. La diversité des procédures d'enregistrement mises en place par les statisticiens locaux aboutit rapidement à une tentative d'harmonisation par le Bureau statistique du Sobež. À partir de la fin de septembre, ce Bureau envoya à tous les comités locaux des Unions situés dans les provinces d'accueil des formulaires-types à renvoyer trois fois par mois. En octobre 1915, l'enregistrement du nombre de réfugiés déboucha sur une initiative plus élaborée dont le but final était de procéder à un recensement général des réfugiés (povsemestnaja perepis' bežencev). Il s'agissait d'identifier le réfugié, par rapport à son lieu d'origine mais aussi dans son contexte familial, social et national. Pour le Bureau statistique du Sobež, l'enregistrement des réfugiés était conçu dans une perspective de rationalisation de leur répartition territoriale. Ainsi, l'identification du réfugié suivait avant tout une logique d'intégration, à la fois économique et culturelle, dans les provinces d'accueil. Le travail fut d'ailleurs mené en étroite corrélation avec le Central'nyj bjuro truda du Sobež qui avait pour tâche de trouver des emplois aux réfugiés dans les provinces d'accueil. En mars 1916, ce Bureau avait établi des contacts avec près de 140 organisations nationales et mis en place également des bureaux locaux de placement dans 56 villes ${ }^{84}$. Enfin, l'analyse du mouvement réfugié relevait également d'une volonté de maintenir l'intégrité des familles, d'où l'importance accordée à la recherche des réfugiés disparus. Cette action, menée par le Bureau d'information du Sobež (Spravočnyj bjuro) et prenant appui sur les données fournies par le Bureau statistique, permit de retrouver, au 1er mars 1916, environ $13 \%$ des réfugiés recensés disparus (soit 14694

83. Vserossijskie zemskij i gorodskoj sojuzy, Otdel po ustrojstvu bežencev, Očerk dejatel'nosti. Moscou, 1916, p. 24-25.

84. Le Central'nyj bjuro truda s'était occupé du placement des réfugiés dans des compagnies ferroviaires, mais aussi dans des usines d'armement, ainsi que pour la construction de tranchées. Astrov, Gronsky, The War and the Russian Government..., p. 237. 
personnes) ${ }^{85}$. Quant à la vaste opération d'enregistrement des réfugiés, elle aboutit, au début de 1916, à la publication d'un premier bilan statistique faisant état de 2678702 réfugiés recensés, dont 2467881 réfugiés pour la seule Russie d'Europe ${ }^{86}$.

L'immense travail statistique fourni par le Sobež, au cours des premiers mois de son existence, prit fin contre sa volonté le 26 novembre 1915, avec la décision adoptée par le Conseil spécial aux réfugiés, sous l'impulsion du ministre de l'Intérieur, de confier la poursuite de l'enregistrement des réfugiés au Comité Tatiana et plus particulièrement à son Département spécial pour l'enregistrement des réfugiés (Osobyj otdel po registracii bežencev) ${ }^{87}$. En activité depuis la mi-septembre, ce Département avait pour principale fonction de recenser et de rechercher les réfugiés disparus par le biais notamment de vastes campagnes d'affichage à travers toute la Russie. Doté d'importants moyens logistiques et financiers et assuré du soutien des autorités gouvernementales et des bureaucraties locales, le Comité Tatiana avait obtenu pour cette opération de meilleurs résultats que le Sobež. L'efficacité reconnue de cette action, mais aussi et surtout le fait que le Département était, de facto, une émanation du Comité central de statistique, incitèrent le ministère de l'Intérieur à vouloir transférer la conduite des opérations de recensement des Unions vers une structure qui lui était proche ${ }^{88}$. Et ce, d'autant plus que les statisticiens du Bureau d'enregistrement du Comité Tatiana avaient spontanément entrepris d'élargir leurs procédures de recherche des réfugiés à l'analyse statistique du mouvement des réfugiés ${ }^{89}$.

Le 6 février 1916, les protestations virulentes du prince L'vov et de M. M. Ščepkin au Conseil spécial aux réfugiés relancèrent la discussion sur la question du recensement des réfugiés. Face à l'unanimité manifestée par les membres du Conseil, y compris par les représentants des principales organisations nationales, pour que le Comité Tatiana constituât le seul organe central d'enregistrement des réfugiés, M. M. Ščepkin déclara qu'il ne s'y opposerait plus ${ }^{90}$. D'autant plus que, selon lui, cette question ne pouvait être résolue que dans le cadre plus général du problème de la reconnaissance par les acteurs étatiques de la dimension nouvelle

85. Vserossijskie zemskij i gorodskoj sojuzy..., Očerk dejatel'nosti, p. 7.

86. Ibid, p. 28. Outre la publication de ces données générales, le rapport a fourni de précieuses informations sur la répartition des réfugiés par sexe, âge, nationalité, distribution entre villes et campagnes.

87. Les deux représentants des Unions étaient absents de cette séance. Ščepkin, Bežency i organizacija pomošči..., p. 39-40.

88. Le directeur du Département spécial aux réfugiés du Comité Tatiana était V. I. Komarnickij, le directeur adjoint du CSK.

89. V. I. Komarnickij, Zapiska o postanovke dela registracii i rozyska bežencev v Osobom otdele Komiteta po registracii bežencev ee imperatorskogo vysočestva velikoj knjažny Tatjany Nikolaevny. RGVIA, f. 13273, op. 1, d. 37,1. 65.

90. Le député polonais de la Douma, H. Svencicki et les représentants du Comité central letton et du Comité central lituanien, S. A. Šilling et Janis Zalitis, intervinrent au Conseil pour marquer leur préférence pour le Comité Tatiana. Žurnal Osobogo soveščanija po ustrojstvu bežencev. RGIA, f. 1322, op. 1, d. 1,1.212-213. 
acquise par les Unions au cours de la guerre. Reconnaissance qui, comme le soulignait Ščepkin, était loin d'être d'actualité. L'éviction définitive des Unions de la question réfugiée (traduite essentiellement par l'absence de financement des programmes menés par le Sobež) aboutit, dans la seconde moitié de l'année 1916, à la fermeture progressive de la plupart de ses services. Cette mise à l'écart s'inscrivait dans un contexte d'hostilité désormais ouvert entre les Unions d'une part, et le nouveau président du Conseil des ministres, B. Šturmer, et la monarchie impériale, d'autre part. Le partenariat entre forces étatiques et forces publiques dans la mobilisation de la Russie dans la guerre, partenariat qui s'était traduit par une participation des Unions aux principaux organes étatiques de régulation de l'économie de guerre, les Conseils spéciaux, devint ainsi illusoire. Au sein même des Unions, l'idée que les actions entreprises par elles-mêmes avaient acquis une dimension «étatique » (gosudarstvennoe) empreignit le discours des responsables. Pour le prince L'vov, un glissement s'était produit au sein de la société civile qui avait fait la preuve, à travers les Unions, de ses capacités «étatiques» (russkaja obščestvennost' stala obščestvennost'ju gosudarstvennoj) ${ }^{91}$. L'action publique avait donc pour vocation non plus de suppléer, mais de remplacer l'action étatique. La campagne contre les Unions, lancée au printemps 1916 par B. Šturmer en accord avec le couple impérial, visait notamment à limiter l'expression de ce discours sur la scène publique. Le décret impérial d'avril 1916 imposait ainsi l'autorisation préalable du ministère de l'Intérieur à la tenue de tout grand rassemblement organisé par les forces civiles, y compris des congrès des représentants des Unions. En décembre 1916, le congrès des Unions fut d'ailleurs interdit et ses participants dispersés par la police.

Avec le déclenchement du premier conflit mondial, le regroupement des selfgouvernements dans des structures fédératives, les Unions, fournit le cadre à un changement d'échelle et à une mutation de l'action publique. Se trouvant face à un espace laissé en partie vacant par un État soumis à des forces centrifuges, les Unions développèrent de multiples stratégies pour occuper cet espace. L'expérience acquise en matière de politique de développement des territoires administrés au niveau local, mais aussi en termes de gestion des grandes crises dans la période qui suivit 1905, constitua le premier mode de légitimation de la montée en puissance des Unions à partir de 1915. À ceci s'ajoute encore l'impact des grandes actions d'assistance développées avec succès par les Unions auprès de l'armée. Ces actions servirent tout d'abord à sceller une forme d'alliance entre les autorités militaires et les Unions. Mais elles nourrirent également le discours de l' « efficacité », $\mathrm{du}$ «caractère organisé » (planomernyj) du travail mené par les Unions face à l'incompétence des autorités étatiques. Comme l'a montré l'exemple de l'implication des Unions en faveur des réfugiés, l'action humanitaire, soumise aux mêmes principes de rationalité, se fondait elle-même dans une politique cherchant à régler de façon globale des situations de crise, à savoir, dans le cas des populations civiles déplacées, la gestion des flux migratoires. Cette conception de plus en plus poli- 
tique de l'action humanitaire ainsi que l'étendue des compétences prises par les Unions au cours de la guerre facilitèrent le glissement de l'idée d'un partenariat entre l'État et la société civile vers l'ambition d'une substitution du premier par la seconde.

En cherchant à se poser comme les représentants d'une obščestvennost' suffisamment mûre pour prendre les commandes de l'État, les responsables des Unions, et le prince L'vov en particulier, avaient toutefois surestimé leurs capacités mobilisatrices des forces civiles. Comme l'a montré William Gleason, historien de l'Union des zemstvos, les Unions étaient soumises à de fortes tensions internes opposant élus et professionnels. Ce clivage résultait du poids croissant pris par ces derniers dans le fonctionnement des Unions et donc de leur aspiration à une meilleure représentation au sein des organes locaux d'auto-gestion ${ }^{92}$. Ajoutons encore que, contrairement au discours du prince L'vov relayé par certains libéraux selon lequel la guerre aurait soudé la société civile, on assistait de fait à une véritable cristallisation de l'espace public entre des forces concurrentes qui se structuraient mais qui ne poursuivaient pas forcément les même buts. Cet éclatement, bien connu dans le cadre de la vie politique, s'appliquait aussi à d'autres sphères de la vie publique. Par exemple, la marginalisation des Unions en matière de politique en faveur des populations réfugiées devait être mise autant sur le compte des autorités gouvernementales que sur celui des grands organismes d'assistance nationaux et caritatifs qui, eux aussi, d'une certaine façon, profitèrent de l'espace vacant laissé par l'État pour s'organiser et se mobiliser. Néanmoins, à la veille des événements de février 1917, les Unions étaient dotées d'une légitimité qui pourrait être définie par une présence, certes inégale, mais néanmoins constante, dans tous les domaines de l'effort de guerre. Cette polyvalence contribua très certainement à faire des responsables des Unions des zemstvos et des villes, des acteurs majeurs du nouvel ordre politique instauré en février 1917.

S'étant substitués à la bureaucratie impériale (les charges de gouverneurs et de gradonačal'niki furent supprimées dès mars 1917) pour la conduite des affaires locales, les zemstvos et les municipalités furent rapidement débordés par l'esprit révolutionnaire qui s'était propagé au sein de leur personnel. Les administrateurs des zemstvos finirent également par incarner l'ordre ancien aux yeux d'une paysannerie aspirant au changement radical. La venue des bolcheviks au pouvoir finit de balayer cette expérience nouvelle de gestion d'un pays en pleine mutation politique et sociale. L'Union des zemstvos fut dissoute fin décembre 1917, celle des villes début janvier. Les zemstvos et les doumas municipales furent supprimées en février $1918^{93}$. Au gré de la formation des armées blanches, certains anciens administrateurs des Unions s'efforcèrent de reconstituer, ici où là, des fédérations des organes d'auto-administration dans le but de participer à l'administration des territoires

92. Gleason, « The All-Russian Union of Zemstvos and World War One », in Emmons, Vucinich, eds., The Zemstvo in Russia..., p. 374-375.

93. William Rosenberg, « The Zemstvo in 1917 and under Bolshevik Rule », in Ibid., p. 405408. Astašev, « Vserossijskij sojuz gorodov », p. 78. 
occupés par les troupes blanches. C'est cette nébuleuse, extrêmement difficile à reconstituer, qui est directement à l'origine, à travers la constitution du Zemgor, de la nouvelle mobilisation en exil de ses acteurs. Héritier d'une longue tradition administrative et ayant acquis avec la guerre une immense expérience en matière de gestion de situations de crise, le Zemgor s'est vu logiquement confier un rôle majeur dans la structuration de l'émigration russe mais il a aussi participé, en tant qu'acteur communautaire, aux grandes orientations du champ humanitaire international de l'après-guerre.

Centre d'études des mondes russe, caucasien et centre-européen EHESS

olga@pichon.info 